\title{
ON THE EQUALITY CASE IN EHRHART'S VOLUME CONJECTURE
}

\author{
BENJAMIN NILL AND ANDREAS PAFFENHOLZ
}

\begin{abstract}
Ehrhart's conjecture proposes a sharp upper bound on the volume of a convex body whose barycenter is its only interior lattice point. Recently, Berman and Berndtsson proved this conjecture for a class of rational polytopes including reflexive polytopes. In particular, they showed that the complex projective space has the maximal anticanonical degree among all toric KählerEinstein Fano manifolds.

In this note, we prove that projective space is the only such toric manifold with maximal degree by proving its corresponding convex-geometric statement. We also discuss a generalized version of Ehrhart's conjecture involving an invariant corresponding to the so-called greatest lower bound on the Ricci curvature.
\end{abstract}

\section{INTRODUCTION}

Minkowski's famous lattice point theorem gives a sharp upper bound on the volume of a centrally-symmetric convex body containing only the origin as a strictly interior lattice point [11]. A convex body $K \subseteq \mathbb{R}^{n}$ is a compact convex set. $K$ is centrally symmetric if $-x \in K$ for all $x \in K$. Two convex bodies $K, K^{\prime}$ in $\mathbb{R}^{n}$ are unimodularly equivalent, if there is an affine lattice automorphism of $\mathbb{Z}^{n}$ mapping $K$ onto $K^{\prime}$. Note that this transformation does not change the volume. Let $\Delta_{n}$ be the $n$-dimensional unimodular simplex with vertices $\mathbf{0}, e_{1}, \ldots, e_{n}$, where $\mathbf{0}=(0, \ldots, 0)$, and $e_{1}, \ldots, e_{n}$ is the standard lattice basis of $\mathbb{Z}^{n}$.

In 1964 Ehrhart [8] posed the following generalization of Minkowski's theorem as a conjecture (see also [3, E13] and [11]).

Conjecture 1.1 (Ehrhart '64). Let $K \subseteq \mathbb{R}^{n}$ be an $n$-dimensional convex body with barycenter $\mathbf{0}$. If $K$ contains only the origin as an interior lattice point, then

$$
\operatorname{vol}(K) \leq(n+1)^{n} / n !
$$

where equality holds if and only if $K$ is unimodularly equivalent to $(n+1) \Delta_{n}$.

Ehrhart proved the upper bound in the conjecture in several special cases, e.g., in dimension $2[6]$ and for simplices [9]. The uniqueness of the equality case is suggested in [8] and proved in dimension 2 [7].

A very good upper bound which is only slightly weaker than the conjectured one can be deduced from a paper of Milman and Pajor. In Corollary 3(2) of [17] they show that $\operatorname{vol}(K) \leq 2^{n} \operatorname{vol}(K \cap(-K))$ for any compact convex body with barycenter in the origin. If additionally the origin is the only lattice point in the interior of $K$, then we can combine this result with Minkowski's first theorem to obtain $\operatorname{vol}(K) \leq 4^{n}$. Let us note that by Stirling's approximation the conjectural upper bound is asymptotically equal to $e^{n+1} / \sqrt{2 \pi n}$.

BN is supported by the US National Science Foundation (DMS 1203162).

AP is supported by the German Research Foundation (DFG SPP 1489). 
For rational polytopes (i.e., polytopes with vertices in $\mathbb{Q}^{n}$ ), there is a natural relation of this conjecture to differential geometry via toric varieties, see e.g., [16, 20]. A polytope $Q$ is a lattice polytope if its vertices are in $\mathbb{Z}^{n}$. Let the origin $\mathbf{0}$ be an interior point of $Q$. In this case, the dual polytope $Q^{*}$ is defined as

$$
Q^{*}:=\left\{x \in \mathbb{R}^{n}:\langle v, x\rangle \geq-1 \text { for all vertices } v \text { of } Q\right\},
$$

where we chose an inner product $\langle\cdot, \cdot\rangle$ on $\mathbb{R}^{n}$ (We remark that the usual convention is to consider dual vector spaces. We use the above notation for the sake of simplicity of this note.) It follows from $Q$ being a lattice polytope that $\mathbf{0}$ is the only interior lattice point of the rational polytope $Q^{*}$. In general, $Q^{*}$ is not a lattice polytope. If it is, then we say that $Q$ and $Q^{*}$ are reflexive polytopes.

For the following, let $Q \subseteq \mathbb{R}^{n}$ be a Fano polytope, i.e., an $n$-dimensional lattice polytope that contains the origin in its interior and whose vertices are primitive (i.e., not multiples of a non-zero lattice point). Then the associated fan over the faces of $Q$ gives rise to a toric Fano variety $X$ (see, e.g., [10] for details of this correspondence). In particular, $X \cong \mathbb{P}^{n}$ if and only if the dual of the associated polytope $Q$ is unimodularly equivalent to $(n+1) \Delta_{n}$. Wang and Zhu [23] (in the nonsingular case) and Berman and Berndtsson [2] (in the singular case) showed that $X$ admits a Kähler-Einstein metric if and only if the barycenter of $Q^{*}$ is 0. Moreover, it is known that the anticanonical degree $c_{1}(X)^{n}$ equals $n ! \operatorname{vol}\left(Q^{*}\right)$. Hence, Ehrhart's conjecture can be phrased in this setting as the problem of finding the maximal degree of a Kähler-Einstein toric Fano variety [20]. Recently, this was solved by Berman and Berndtsson [2, Thm.1.2]:

Theorem 1.2 (Berman, Berndtsson '12). Let $X$ be an $n$-dimensional toric Fano variety which admits a (singular) Kähler-Einstein metric. Then its first Chern class $c_{1}(X)$ satisfies the following upper bound:

$$
c_{1}(X)^{n} \leq(n+1)^{n}
$$

The motivation of this note is to clarify the equality case:

Proposition 1.3. In the situation of Theorem 1.2, $c_{1}(X)^{n}=(n+1)^{n}$ if and only if $X \cong \mathbb{P}^{n}$.

This is a special case of the following main result.

Theorem 1.4. The conclusion of Conjecture 1.1 holds for any $n$-dimensional convex body $K \subseteq \mathbb{R}^{n}$ with barycenter $\mathbf{0}$ that is contained in the dual polytope of a lattice polytope.

The proof relies on the convex-geometric part of the argument in [2] which Berman and Berndtsson attribute to Bo'az Klartag. The only novel part is the treatment of the equality case. Note that the condition in the theorem is equivalent to the condition that the dual of $K$ contains a full-dimensional lattice polytope having the origin in its interior. Here is an example of a convex body that does not satisfy this condition. Let $K:=\operatorname{conv}( \pm(3 / 2,1 / 4), \pm(3 / 2,5 / 4))$. Then $K$ is a convex body whose only interior lattice point is $\mathbf{0}$. Its dual is $K^{*}=\operatorname{conv}\left( \pm(1,-2), \pm\left(\frac{2}{3}, 0\right)\right)$, and the only lattice points in $K^{*}$ are $\mathbf{0}$ and $\pm(1,-2)$.

Theorem 1.4 generalizes Corollary 1.4 in [2]. In particular, Ehrhart's conjecture holds for all reflexive polytopes with barycenter $\mathbf{0}$. This huge class of lattice polytopes is of importance in string theory since they give rise to many examples of mirror-symmetric Calabi-Yau manifolds [1, 18, 13, 14]. 
Organization of the paper: Section 2 contains the proof of Theorem 1.4. In Section 3 we discuss a variant of Conjecture 1.1 regarding the so-called greatest lower bound on the Ricci curvature.

Acknowledgments: We would like to thank Robert Berman and Xiaodong Wang for their interest and for telling us about the generalized version of Ehrhart's conjecture. We thank the referee for several suggestions to improve the paper. In particular, he pointed us to the result of Milman and Pajor mentioned above and its relation to Ehrhart's conjecture. We are especially grateful to Stanislaw Szarek for providing the alternative proof in Remark 2.3 and publishing his proof of Grünbaum's inequality as a courtesy to the readers.

\section{Proof of Theorem 1.4}

We may assume $n \geq 2$. Let $\mathbf{0}$ be the origin in $\mathbb{R}^{n}$ and $\mathbf{1}:=(1, \ldots, 1)$. We follow the approach in the proof of Corollary 1.4 and Remark 3.2 in [2]. A key ingredient of the proof is Grünbaum's inequality [12]. For any convex body $K \subseteq \mathbb{R}^{n}$ and any closed half-space $H$ that contains the barycenter of $K$ it states that

$$
\operatorname{vol}(K \cap H) \geq\left(\frac{n}{n+1}\right)^{n} \operatorname{vol}(K) .
$$

Now let $K \subseteq \mathbb{R}^{n}$ be an $n$-dimensional convex body with barycenter $\mathbf{0}$ that is contained in $P:=Q^{*}$ for a lattice polytope $Q$ containing $\mathbf{0}$ in its interior. Fix a vertex $v$ of $P$. The facets $F$ of $P$ are in one-to-one correspondence with the vertices $l_{F}$ of $Q$. Choose facets $F_{1}, \ldots, F_{n}$ containing $v$ such that $l_{F_{1}}, \ldots, l_{F_{n}}$ span $\mathbb{R}^{n}$. We consider the invertible affine-linear map

$$
\begin{aligned}
\phi: \mathbb{R}^{n} & \longrightarrow \mathbb{R}^{n} \\
x & \longmapsto\left(\left\langle l_{F_{1}}, x\right\rangle+1, \ldots,\left\langle l_{F_{n}}, x\right\rangle+1\right) .
\end{aligned}
$$

By construction, $\phi$ maps the polytope $P$ into the non-negative orthant $\mathbb{R}_{\geq 0}^{n}$. Since $l_{F_{1}}, \ldots, l_{F_{n}}$ are lattice points we have $|\operatorname{det}(\phi)| \geq 1$. In particular,

$$
\operatorname{vol}(K) \leq|\operatorname{det}(\phi)| \operatorname{vol}(K)=\operatorname{vol}(\phi(K)) .
$$

The barycenter of $\phi(K)$ is the image of the barycenter of $K$, so $b:=\phi(\mathbf{0})=\mathbf{1}$. Consider $\eta:=\mathbf{1}$ as a linear functional on $\mathbb{R}^{n}$. Then $\eta^{-}:=\left\{x \in \mathbb{R}^{n}:\langle\eta, x\rangle \leq n\right\}$ is an affine half-space containing the origin in its interior and $b$ on the boundary. Using $\phi(K) \subseteq \phi(P) \subseteq \mathbb{R}_{\geq 0}^{n}$, we get

$$
\begin{aligned}
\left(\frac{n}{n+1}\right)^{n} \operatorname{vol}(\phi(K)) & \leq \operatorname{vol}\left(\phi(K) \cap \eta^{-}\right) \leq \operatorname{vol}\left(\phi(P) \cap \eta^{-}\right) \\
& \leq \operatorname{vol}\left(\mathbb{R}_{\geq 0}^{n} \cap \eta^{-}\right)=\operatorname{vol}\left(n \Delta_{n}\right)=\frac{n^{n}}{n !},
\end{aligned}
$$

where the first inequality follows from (1). Combining this with (2) implies the desired inequality $\operatorname{vol}(K) \leq \operatorname{vol}(\phi(K)) \leq(n+1)^{n} / n !$.

Now, let us assume that $\operatorname{vol}(K)=(n+1)^{n} / n$ !. This implies that all inequalities in (2) and (3) are in fact equalities. The equations in (3) imply $\mathbb{R}_{\geq 0}^{n} \cap \eta^{-}=\phi(K) \cap \eta^{-}$. Hence, $\phi(v)=\mathbf{0} \in \mathbb{R}_{\geq 0}^{n} \cap \eta^{-}$yields $v \in K$. Since $v$ was chosen arbitrarily we get $K=P$. Moreover, equality in (2) implies that $|\operatorname{det}(\phi)|=1$, so $\phi$ is an affine lattice transformation of $\mathbb{Z}^{n}$. Thus, $P$ and $\phi(P)$ are unimodularly equivalent. In particular, since $\mathbf{0}$ is a lattice point, also $v$ is a lattice point. Again, since $v$ was arbitrary, $P$ is a lattice polytope, and therefore reflexive (recall that by assumption $P^{*}=Q$ is a lattice polytope). 
We will use the following result on reflexive polytopes contained in the paper [19] of the first author:

Lemma 2.1. Let $S$ be a reflexive polytope with barycenter in the origin. If $m$ is a relatively interior lattice point of a facet of $S$, then $-m$ is again in the relative interior of a facet of $S$.

Proof. This is precisely the implication from i. to (a) in part (2) of Theorem 5.2 in [19]. In that paper $P$ being semisimple means that the set of roots is centrallysymmetric; and the set of roots is the precisely the set of lattice points in the relative interior of facets of $P$, see Definition 4.1 in [19] and the paragraph before.

It remains to consider the following situation: We have an $n$-dimensional polytope $R$ (which is the polytope $\phi(P)$ above) with $\operatorname{vol}(R)=\operatorname{vol}\left((n+1) \Delta_{n}\right)$ such that

(1) the barycenter $b_{R}$ equals 1.

(2) $n \Delta_{n} \subseteq R$ and they share the vertex cone at $\mathbf{0}$. Thus $e_{1}, \ldots, e_{n}$ are primitive inner facet normals.

(3) $R-\mathbf{1}$ is a reflexive polytope.

Lemma 2.2. In this situation, we also have the following condition:

(4) for any $i=1, \ldots, n$, the point $\mathbf{1}+e_{i}$ is contained in the relative interior of a facet of $R$.

Proof. We may assume $i=1$. Since $n \Delta_{n} \subseteq R$, we see that $\mathbf{1}-e_{1}$ is in the relative interior of the facet $F:=\left\{x \in R:\left\langle e_{1}, x\right\rangle=0\right\}$. Hence, we can apply Lemma 2.1 to $R-\mathbf{1}$ and $m=\left(\mathbf{1}-e_{1}\right)-\mathbf{1}=-e_{1}$ to see that $e_{1}$ is in the relative interior of a facet of $R-\mathbf{1}$. This proves the claim.

We will show that $R=(n+1) \Delta_{n}$. For $i=1, \ldots, n$, let us denote by $G_{i}$ the unique facet of $R$ containing $m_{i}:=\mathbf{1}+e_{i}$. Furthermore, let $u_{i}$ be the primitive outer normal of $G_{i}$. Translating a polytope does not change the normal fan, so, as $R-\mathbf{1}$ is reflexive, we obtain

(4) $\left\langle u_{i}, m_{i}-\mathbf{1}\right\rangle=\left\langle u_{i}, e_{i}\right\rangle=1$ and $\left\langle u_{i}, m_{j}-\mathbf{1}\right\rangle=\left\langle u_{i}, e_{j}\right\rangle \leq 1$ for $j \neq i$

Let $u_{1}:=\left(c_{1}, \ldots, c_{n}\right)$. Then (4) implies $c_{1}=1$ and $c_{j} \leq 1$ for $j=2, \ldots, n$. If $c_{j}=1$ for some $j \geq 2$, then $m_{j} \in G_{1}$, and thus $G_{j}=G_{1}$.

By assumption we have $n \Delta_{n} \subseteq R$, so $\left\langle u_{1}, n e_{1}-\mathbf{1}\right\rangle \leq 1$. Thus, for any $j \geq 2$

$$
\begin{aligned}
n & =\left\langle u_{1}, n \cdot e_{1}\right\rangle \leq\left\langle u_{1}, \mathbf{1}\right\rangle+1=2+c_{2}+c_{3}+\cdots+c_{n} \\
& \leq 1+c_{j}+(n-1)=n+c_{j} .
\end{aligned}
$$

This implies that $c_{j} \geq 0$, so $c_{j} \in\{0,1\}$ for $j=2, \ldots, n$. Furthermore, if $c_{j}=0$ for some $j$, then $c_{k}=1$ for all $k$ except $j$.

Now assume that indeed there is some $j$ with $c_{j}=0$, say $j=2$. Then $u_{1}=1-e_{2}$. We have observed above that $c_{j}=1$ implies $G_{j}=G_{1}$, hence $G_{1}=G_{3}=\cdots=G_{n}$. We consider $u_{2}:=\left(d_{1}, \ldots, d_{n}\right)$. Similarly as for $u_{1}$ we obtain $d_{2}=1$ and $d_{j} \in\{0,1\}$ for $j \neq 2$. Since $m_{2}=\mathbf{1}+e_{2} \notin G_{1}$ (because of $\left\langle u_{1}, m_{2}-\mathbf{1}\right\rangle=0<1$ ), we have $G_{2} \neq G_{j}$ for $j \in\{1,3, \ldots, n\}$. Hence, again by the above remark, we must have $d_{k} \neq 1$ for any $k=1,3, \ldots, n$. Therefore, $u_{2}=e_{2}$, so $\left\langle u_{1}, m_{1}\right\rangle=n$ and $\left\langle u_{2}, m_{2}\right\rangle=2$ yields

$$
R \subseteq\left\{x \in \mathbb{R}_{\geq 0}^{n}:\left\langle\mathbf{1}-e_{2}, x\right\rangle \leq n,\left\langle e_{2}, x\right\rangle \leq 2\right\} .
$$


Here, the right polytope is unimodularly equivalent to $\left(n \Delta_{n-1}\right) \times[0,2]$. Therefore,

$$
\frac{(n+1)^{n}}{n !}=\operatorname{vol}(R) \leq \frac{2 n^{n-1}}{(n-1) !},
$$

which implies $(n+1)^{n} \leq 2 n^{n}$, a contradiction for $n \geq 2$.

Therefore, $c_{j}=1$ for $j=2, \ldots, n$. Hence, $u_{1}=(1, \ldots, 1)$. So,

$$
R \subseteq\left\{x \in \mathbb{R}_{\geq 0}^{n}:\langle\mathbf{1}, x\rangle \leq n+1\right\}=(n+1) \Delta_{n} .
$$

Since both polytopes have the same volume, we get equality.

Remark 2.3. As was pointed out to us by Szarek after this paper has appeared as a preprint, the proof can be substantially simplified by using a characterization of the equality case in Grünbaum's inequality. In the notation of the proof: inequality (1) holds with equality if and only if $K$ is a pyramid over a base spanning an affine hyperplane parallel to $h$, where $h$ is the affine hyperplane bounding the half-space $H$.

Here is how one applies this result in the equality case. Let $R=\phi(P)$ again with $\operatorname{vol}(R)=\operatorname{vol}\left((n+1) \Delta_{n}\right)$, barycenter $b_{R}=1$, and $n \Delta_{n} \subseteq R$. From the previous result we deduce that $R$ is a pyramid with apex $\mathbf{0}$ and whose base facet has outer facet normal 1 . Since the $n$ coordinate planes are supporting facet hyperplanes of $R$, this implies that $R$ must be a simplex of the form $\lambda \Delta_{n}$ for some $\lambda \geq n$. From $\operatorname{vol}(R)=\operatorname{vol}\left((n+1) \Delta_{n}\right)$ we get $\lambda=n+1$, as desired.

At the time this paper was published as a preprint, the proof of the equality characterization of Grünbaum's inequality was not available in the literature. Morever, the main result in Grünbaum's original paper [12] is stated slightly differently, and so a characterization of the equality case in that setting wouldn't be directly applicable to our situation. In 2003, Szarek found an alternative proof of Grünbaum's inequality that allows to deal more directly with the case of equality. He made his proof now publicly available [21].

\section{Generalized Ehrhart's Conjecture and greatest lower bound on THE RiCCI CURVATURE}

For a general Fano manifold $X$ one can define

$$
R(X):=\sup \left(t \in[0,1]: \text { Ric } \omega \geq t \omega \text { for all Kähler metrics } \omega \in c_{1}(X)\right),
$$

giving a "greatest lower bound on the Ricci curvature". See [15, 22] for background and motivation. In Theorem 2.9 of [2], Berman and Berndtsson gave (in more generality) the following generalization of Theorem 1.2 in the case that there is no Kähler-Einstein metric:

Theorem 3.1 (Berman, Berndtsson '12). Let X be a toric Fano manifold. Then the first Chern class $c_{1}(X)$ satisfies the following upper bound

$$
c_{1}(X)^{n} \leq\left(\frac{n+1}{R(X)}\right)^{n}
$$

As a convex-geometric analogue, $\mathrm{Li}[15]$ introduced the following invariant for a convex body $K$. 
Definition 3.2. Let $K \subseteq \mathbb{R}^{n}$ be an $n$-dimensional convex body containing $\mathbf{0}$ in its interior. Let $b_{K}$ be its barycenter. If $b_{K}=\mathbf{0}$, then we define $R(K):=1$. Otherwise, let $x_{K}$ be the intersection of $b_{K}-\mathbb{R}_{\geq 0} b_{K}$ with the boundary of $K$. Then $R(K)$ is defined as the distance of $x_{K}$ from 0 divided by the distance of $x_{K}$ from $b_{K}$. In particular, $R(K)<1$ in this case.

Let $Q$ be an $n$-dimensional Fano polytope with associated toric variety $X_{Q}$. In this case Li [15] showed that for smooth $X_{Q}$ the two definitions of $R$ coincide, i.e. $R(X)=R\left(Q^{*}\right)$.

Taking this invariant $R(K)$ into account, Xiaodong Wang suggested a natural generalization of Ehrhart's conjecture for convex bodies with arbitrary barycenter.

Conjecture 3.3. Let $K \subseteq \mathbb{R}^{n}$ be an $n$-dimensional convex body. If $K$ contains only the origin as an interior lattice point, then

$$
\operatorname{vol}(K) \leq \frac{(n+1)^{n}}{n ! R(K)^{n}}
$$

where equality holds if and only if $K$ is unimodularly equivalent to $(n+1) \Delta_{n}$.

We would like to remark that this apparent generalization is actually equivalent to Ehrhart's conjecture.

Proposition 3.4. Conjecture 3.3 is equivalent to Conjecture 1.1.

Proof. Let $K \subseteq \mathbb{R}^{n}$ be an $n$-dimensional convex body such that $K$ contains only the origin as an interior lattice point. Let $r:=R(K)<1$, and $b_{K}$ the barycenter of $K$.

We define $K^{\prime}:=r\left(K-b_{K}\right)$. We claim $K^{\prime} \subseteq K$. For this, let $u \in \mathbb{R}^{n}, c \in \mathbb{R}$ such that $\langle u, x\rangle \leq c$ for any $x \in K$. Then $\left\langle u, r\left(x-b_{K}\right)\right\rangle \leq r\left(c-\left\langle u, b_{K}\right\rangle\right)$. It suffices to show that $r\left(c-\left\langle u, b_{K}\right\rangle\right) \leq c$. However, this is equivalent to $\left\langle u,(r /(r-1)) b_{K}\right\rangle \leq c$ which holds by our assumption: $(r /(r-1)) b_{K}=x_{K} \in K$.

Therefore, $K^{\prime}$ is an $n$-dimensional convex body with barycenter $\mathbf{0}$ which contains no other interior lattice points. Since $\operatorname{vol}\left(K^{\prime}\right)=r^{n} \operatorname{vol}(K)$, we have $\operatorname{vol}\left(K^{\prime}\right) \leq$ $(n+1)^{n} / n$ ! if and only if $\operatorname{vol}(K) \leq(n+1)^{n} /\left(n ! r^{n}\right)$. It remains to consider the equality case. Assume $K^{\prime}=(n+1) \Delta_{n}$. Since $K \supseteq K^{\prime}$ and any facet of $K^{\prime}$ contains a lattice point in its relative interior, we have $K=K^{\prime}$.

From the proof we get the following generalization of Theorem 1.4.

Corollary 3.5. The conclusion of Conjecture 3.3 holds for any n-dimensional convex body $K \subseteq \mathbb{R}^{n}$ that is contained in the dual polytope of a lattice polytope.

In particular, this implies a convex-geometric proof of Theorem 3.1. The following consequence seems to be new:

Corollary 3.6. In the situation of Theorem $3.1, c_{1}(X)^{n}=\left(\frac{n+1}{R(X)}\right)^{n}$ if and only if $X \cong \mathbb{P}^{n}$.

Proof. Recall that $c_{1}(X)^{n}=n ! \operatorname{vol}\left(Q^{*}\right)$ and $R(X)=R\left(Q^{*}\right)$. Hence, if equality in Theorem 3.1 holds, then the previous corollary implies that $Q^{*}$ is unimodularly equivalent to $(n+1) \Delta_{n}$, thus $X \cong \mathbb{P}^{n}$. On the other hand, if $X \cong \mathbb{P}^{n}$, then $c_{1}(X)^{n}=(n+1)^{n}$ and $R(X)=1$.

Finally, let us remark that Debarre showed in [4] on p.139 that for a general toric Fano $n$-fold $X$ there is no polynomial bound on $\sqrt[n]{c_{1}(X)^{n}}$. In particular, there are examples of toric Fano $n$-folds $X_{n}$ such that $R\left(X_{n}\right) \rightarrow 0$ for $n \rightarrow \infty$. 


\section{REFERENCES}

[1] V.V.Batyrev, Dual polyhedra and mirror symmetry for Calabi-Yau hypersurfaces in toric varieties, J. Algebr. Geom. 3 (1994), 493-535.

[2] R.J. Berman, B. Berndtsson, The volume of Kähler-Einstein Fano varieties and convex bodies, Preprint, arXiv:1204.1308, 2012.

[3] H.T. Croft, K.J. Falconer, R.K. Guy, Unsolved problems in geometry, Problem Books in Mathematics 2, New York, NY, Springer, 1991.

[4] O. Debarre, Higher-dimensional algebraic geometry, Universitext, New York, NY, Springer, 2001.

[5] O. Debarre, Fano varieties, In: K.jun. Böröczky (ed.) et al., Higher dimensional varieties and rational points, Lectures of the summer school and conference (Budapest, Hungary, 2001), Springer-Verlag, Bolyai Society Mathematical Studies 12, 93-132, 2003.

[6] E. Ehrhart, Une généralisation du théorème de Minkowski, C. R. Acad. Sci. Paris 240 (1955), 483-485.

[7] E. Ehrhart, Propriétés arithmogéométriques des polygones, C. R. Acad. Sci. Paris 241 (1955), 686-689.

[8] E. Ehrhart, Une généralisation probable du théorème fondamental de Minkowski, C. R. Acad. Sci. Paris 258 (1964), 4885-4887.

[9] E. Ehrhart, Volume réticulaire critique d'un simplexe, J. Reine Angew. Math. 305 (1978), $218-220$.

[10] W. Fulton, Introduction to Toric Varieties, Princeton University Press, 1993

[11] P. Gritzmann, J.M. Wills, Lattice points, in: Handbook of convex geometry, 765-797, NorthHolland, Amsterdam, 1993.

[12] B. Grünbaum, Partitions of mass-distributions and of convex bodies by hyperplanes, Pacific J. Math. 10 (1960), 12571261.

[13] M. Kreuzer, H. Skarke, Classification of reflexive polyhedra in three dimensions, Adv. Theor. Math. Phys. 2 (1998), 853-871.

[14] M. Kreuzer, H. Skarke, Complete classification of reflexive polyhedra in four dimensions, Adv. Theor. Math. Phys. 4 (2000), 1209-1230.

[15] C. Li, Greatest lower bounds on Ricci curvature for toric Fano manifolds, Adv. Math. 226 (2011), 4921-4932.

[16] T. Mabuchi, Einstein-Kähler forms, Futaki invariants and convex geometry on toric Fano varieties, Osaka Journal of Mathematics 24 (1987), 705-737.

[17] V.D. Milman and A. Pajor, Entropy and asymptotic geometry of non-symmetric convex bodies, Adv. Math. 152, no. 2 (2000), 314-335.

[18] B. Nill, Gorenstein toric Fano varieties, Manuscr. Math. 116 (2005), 183-210.

[19] B. Nill, Complete toric varieties with reductive automorphism group, Mathematische Zeitschrift 252 (2006), 767-786.

[20] B. Nill, A. Paffenholz, Examples of non-symmetric Kähler-Einstein toric Fano manifolds, Beitr. Alg. Geom. 52 (2011), 279-304.

[21] S. Szarek, On measures of symmetry and floating bodies, Eprint, arXiv:1302.2076, 2013.

[22] G. Szkelyhidi, Greatest lower bounds on the Ricci curvature of Fano manifolds, Compos. Math. 147 (2011), 319-331.

[23] X. Wang, X. Zhu, Kähler-Ricci solitons on toric manifolds with positive first Chern class, Advances in Mathematics 188 (2004), 87-103.

Benjamin Nill, Case Western Reserve University, Cleveland, OH, USA

E-mail address: benjamin.nill@case.edu

Andreas Paffenholz, TU Darmstadt, Darmstadt, Germany

E-mail address: paffenholz@mathematik.tu-darmstadt.de 